\title{
Effects of Small-Dose S-Ketamine On Anesthesia- Induced Atelectasis In Patients Undergoing General Anesthesia Accessed By Lung Ultrasound: Study Protocol For A Randomized, Double-Blinded Controlled Trial
}

\section{Di Zhang}

Beijing Tiantan Hospital

Yi Liang

Beijing Tiantan Hospital

Di Bao

Beijing Tiantan Hospital

Wei Xiong

Beijing Tiantan Hospital

Lu Li

Beijing Tiantan Hospital

Yaxin Wang

Beijing Tiantan Hospital

Bin Liu

Beijing Tiantan Hospital

Xu Jin ( $\nabla$ xiehe2000@163.com )

Beijing Tiantan Hospital https://orcid.org/0000-0001-5944-0930

\section{Study protocol}

Keywords: Atelectasis, S-ketamine, Ultrasound, Randomized Controlled Trial, Protocol

Posted Date: June 28th, 2021

DOI: https://doi.org/10.21203/rs.3.rs-374038/v1

License: (a) (i) This work is licensed under a Creative Commons Attribution 4.0 International License. Read Full License 


\section{Abstract}

Background: Aelectasis after anesthesia induction in most patients undergoing general anesthesia may lead to postoperative pulmonary complications (PPCs) and affect postoperative outcomes. However, there is still no existing effective method used for the prevention of perioperative atelectasis. S-ketamine may prevent atelectasis due to airway smooth muscle relaxation and anti-inflammatory effects. Lung ultrasound is a portable and reliable bedside imaging technology for diagnosing anesthesia-induced atelectasis. In this study, we aim to assess whether a small dose of S-ketamine can reduce the incidence of atelectasis after intubation under general anesthesia using lung ultrasound, in order to prevent the early formation of perioperative atelectasis and PPCs.

Methods: This is a single-institution, prospective, randomized controlled, parallel grouping, and double-blind study. From October 2020 to March 2022, 100 patients (18-60 years old) scheduled for elective surgery will be recruited from Beijing Tiantan Hospital, Capital Medical University, and randomly assigned to the Sketamine group (Group $\mathrm{S}$ ) and the normal saline group (Group $\mathrm{N}$ ) at a ratio of 1:1. The label-masked agents will be administered 5 min before induction, and all patients will undergo a standardized general anesthesia protocol. Related data will be collected at three-time points: after radial artery puncture (T1), 15 min after tracheal intubation (T2), and before extubation (T3). The primary outcome will be the total lung ultrasound scores (LUS) at T2. Secondary outcomes will include LUS in six chest regions at T2, total LUS at T3, arterial blood-gas analysis results $\left(\mathrm{PaCO}_{2}, \mathrm{PaO}_{2}\right)$ and $\mathrm{PaO}_{2} / \mathrm{FiO}_{2}$ at $\mathrm{T} 2$ and $\mathrm{T} 3$, plateau pressure $\left(\mathrm{P}_{\text {plat }}\right)$ and dynamic lung compliance (Cdyn) at T2 and T3. The incidence of postoperative complications associated with Sketamine and PPCs at $2 \mathrm{~h}$ and $24 \mathrm{~h}$ after surgery will be recorded.

Discussion: This trial aims to explore whether a simple and feasible application of S-ketamine before the induction of general anesthesia can prevent atelectasis. The results of this study may provide new ideas and direct clinical evidence for the prevention and treatment of perioperative pulmonary complications during anesthesia.

Trial registration: ClinicalTrials.gov identifier: NCT04745286.

\section{Background}

Atelectasis refers to the complete or partial collapse of the entire lung or an area of the lung. Previous studies have reported that the incidence of pulmonary atelectasis during general anesthesia is as high as $85-90 \%[1]$. It usually appears a few minutes after the application of $100 \%$ oxygen for pre-oxygenation and induction and continues until after surgery[2,3]. Atelectasis plays an important role in the intrapulmonary shunt increase [4], gas exchange abnormalities, hypoxemia, and compliance decrease. These are associated with postoperative pulmonary complications (PPCs). It seriously affects the postoperative outcome in surgical patients [5-8].

The mechanism of perioperative atelectasis remains unclear. At present, the following three mechanisms have been mainly considered: compressive atelectasis, gas absorption, and alveolar surfactant destruction[3, 9-14]. The mechanism of atelectasis after anesthesia induction is mainly considered to be related to small airway closure and gas absorption in pre-oxygenated alveoli [15]. 
Ketamine and its S-isomer have been widely used in clinical anesthesia, especially in patients with sepsis or cardiovascular instability[16]. Ketamine and its S-isomer achieve anesthesia and analgesia primarily through non-competitive antagonism at N-methyl-d-aspartate receptors. For the different effects of other anesthetics, S-ketamine has the following effects on airway smooth muscle and alveoli, which may prevent atelectasis after anesthesia induction:

(1) Relaxing the bronchial smooth muscles to reduce gas absorption: atelectasis is more likely to occur in lung tissue with decreased ventilation/perfusion (VA/Q) due to insufficient ventilation or increased intrapulmonary shunt[12]. After anesthesia induction, the closure of small airways can decrease ventilation of dependent lung regions to become 'low $\mathrm{V}_{\mathrm{A}} / \mathrm{Q}$ ' units, which increases the gradual absorption of gas from the alveoli to distal residual lung units through the capillary network. Studies have shown that ketamine can relax bronchial smooth muscles in order to alleviate the closure of small airways. Thereby, significantly reducing ventilation resistance [17-19]. This effect is especially beneficial for patients with asthma and bronchospasm.

(2) Inhibit inflammation to reduce the destruction of alveolar surfactant: Studies have shown that anesthesia can suppress the stabilizing function of alveolar surfactant. Furthermore, the shearing force produced by repetitive opening and closing of the alveoli during mechanical ventilation may induce lung injury and deactivation of the alveolar surfactant[20]. Previous studies have shown that ketamine has a unique pleiotropic anti-inflammatory effect[3, 17-19, 21-24]. This anti-inflammatory effect can significantly attenuate ventilator-associated lung injury and direct damage to type II alveolar cells caused by inflammation. Thereby, reducing the loss of the active component of alveolar surfactant, including SP-A and phospholipids. Ketamine prevents exacerbated pro-inflammatory reactions and extension of local inflammation mainly by inducing anti-inflammatory mediators such as adenosine[25]. These anti = inflammatory mediators inhibit the activation of nuclear factor NF-KB and repress the release of proinflammatory cytokines such as TNF-a, IL-6, and IL-8 [19, 25, 26]. In addition, it suppresses the activation and recruitment of leukocytes and precipitates the apoptosis of inflammatory cells in the lungs[27-29]. Among all the detection methods of pulmonary disease, lung ultrasound, as a bedside, non-invasive, diagnostic tool, has been increasingly used in the assessment of atelectasis during surgery. Furthermore, it has advantages such as convenience, rapidity, and it is radiation-free [30-33]. It has a reliable sensitivity of $93 \%$ and specificity of $100 \%[34]$.

Previous studies on atelectasis during anesthesia mostly focused on the influence of respiratory parameters such as tidal volume, respiratory rate, inhaled oxygen concentration, positive end-expiratory pressure (PEEP), and the effects of manipulation or mechanical expansion on postoperative atelectasis[8, 35-37]. To date, there is only a little evidence available for pre-treatment with medicine for atelectasis during general anesthesia. This trial aimed to evaluate the effect of low-dose S-ketamine on atelectasis after tracheal intubation under general anesthesia, Furthermore, to evaluate whether small-dose S-ketamine can prevent perioperative atelectasis. The reasons for choosing lung ultrasound scores (LUS) at 15 min after endotracheal intubation as the primary outcome are as follows: (1) previous studies showed that atelectasis has already occurred at this moment after induction and pre-oxygenation with $100 \%$ oxygen[38]; (2) this period is within the active duration of a single small dose of S-ketamine, and the effect of operation time and 
extubation on atelectasis can be excluded; (3) lung ultrasound can detect atelectasis in real-time, and LUS can accurately reflect the pulmonary state.

\section{Methods}

\section{Trial design and study setting}

This is a single-center, prospective, double-blind, parallel-grouping, randomized controlled trial conducted at Beijing Tiantan Hospital, Capital Medical University, from October 2020 to December 2022. This study was approved by the Institutional Review Board of the Capital Medical University Hospital Institutional Review Board (IRB) and registered at clinicaltrials.gov (Trial Registration Number: NCT04745286). The patient flow diagram of the study is presented in Fig. 1, and the trial schedule is shown in Table 1.

\section{Eligibility}

The trial investigator will identify consecutive eligible patients according to the inclusion and exclusion criteria on the day before the operation. When the patients meet the eligibility criteria, the investigators will explain the study plan and relevant information to the participants.

Inclusion criteria:

(1) Age of $18-60$ years;

(2) American Society of Anesthesiologists (ASA) physical status of I-I;;

(3) Patients scheduled for urinary, obstetrics, and gynecology surgery in the supine or lithotomy position under general anesthesia with expected operation time of more than $1 \mathrm{~h}$.

(4) Patients and their authorized surrogates will sign an informed consent form.

Exclusion criteria:

(1) Patients in whom the application of lung ultrasound is difficult, such as patients with chest fractures or history of surgical.

(2) Patients with a history of upper respiratory tract infection one month before surgery or a history of smoking more than 6 packs/year.

(3) When the preoperative chest X-ray or CT results are abnormal, including atelectasis, pneumothorax, pleural effusion, or pneumonia.

(4) $\mathrm{BMl}>30 \mathrm{~kg} / \mathrm{m}^{2}$;

(5) Expected difficulties in intubation or mask ventilation;

(6) Patients with a significant increase in intraocular pressure and intracranial pressure before surgery;

(7) Patients who are allergic to S-ketamine, propofol, and opioids.

(8) Patients with a history of psychiatric disorders. 


\section{Interventions, allocation, and randomization}

The study investigator will be trained to implement standardized intervention methods. An independent statistician will perform randomization using a computer random number list generated by Stata software version 15.1. Participants will be randomly assigned to either the S-ketamine group (group S) or the normal saline group (group N), and the allocation ratio will be 1:1. Group allocations will be sealed in sequentially numbered opaque envelopes. An independent study researcher who is not involved in anesthesia management or data collection will obtain randomized allocation information and prepare the study agents in the opaque 5-ml syringes before surgery. The blinded anesthesiologists in charge will use the study agents during anesthesia induction to complete the intervention and record the intraoperative data. Two well-trained anesthesiologists will perform lung ultrasound assessments and record LUS at relevant time points. Blinded and trained research assistants will perform the postoperative follow-up $24 \mathrm{~h}$ after the operation to record relevant data. All deviations or missing data will also be recorded. In this study, anesthesiologists in charge, patients, lung ultrasound assessors, and follow-up staff will be blinded to the randomized information. After all the results are completely analyzed, the blinding will be stopped.

\section{Criteria for discontinuing or modifying the allocated interventions}

The assignment will not be modified, however, the study will be terminated in the following situations: (1) intraoperative serious adverse events, such as hemorrhage, shock, and malignant hyperthermia; (2) without anticipated difficult airway or failure to extubation after surgery.

\section{Data collection and management}

All the study-related data of recruited patients will be recorded in an electronic storage system. These data will include baseline information, intraoperative and postoperative follow-up information, and lung ultrasound-related data. The data of each patient will be entered into the EpiData database using a dual data entry system. The data will not be disclosed to other researchers until the study is completed. An inspector will regularly check and revises these records to ensure data quality.

\section{Anesthesia and analgesia}

Standard monitoring of non-invasive blood pressure (BP), heart rate (HR), and pulse oxygen saturation $\left(\mathrm{SpO}_{2}\right)$ will be commenced upon the patient's arrival at the operating room. Radial artery puncture will be performed under local anesthesia to perform blood gas sampling and invasive arterial blood pressure (ABP) monitoring. Patients will receive midazolam $2 \mathrm{mg}$ intravenously as a premedication $5 \mathrm{~min}$ before induction. Anesthesia will be induced by preoxygenation with $100 \%$ oxygen inhalation for 3 min via a face mask. This will be followed by an intravenous administration of sufentanil $0.3-0.5 . \mu \mathrm{g} \cdot \mathrm{kg}^{-1}$, propofol $1.5-2.5 \mathrm{mg} \cdot \mathrm{kg}$ 1 , and rocuronium $0.6-1.0 \mathrm{mg} \cdot \mathrm{kg}^{-1}$. After spontaneous breathing disappears, the volume control mode will be used to control the patient's breathing through the mask for $2 \mathrm{~min}$, with a tidal volume of $6-8 \mathrm{~mL} \cdot \mathrm{kg}--1$ (predicted body weight without PEEP), ventilatory frequency of $12 \mathrm{bpm}$, and $100 \%$ oxygen concentration. It takes approximately 5-7 min from pre-oxygenation to start intubation, and then intubate with a suitably sized tracheal tube with a cuff. All patients will receive mechanical ventilation with a $\mathrm{FiO}_{2}$ of 0.4 , and an 
inspiratory-to-expiratory ratio of 1:1.5-2. The ventilatory parameters will be adjusted to maintain the end-tidal carbon dioxide partial pressure $\left(\mathrm{P}_{\mathrm{ET}} \mathrm{CO}_{2}\right)$ within $35-40 \mathrm{mmHg}$ and airway pressure not exceeding $25 \mathrm{~cm} \mathrm{H}_{2} \mathrm{O}$.

Anesthesia will be maintained with continuous intravenous infusion of remifentanil $0.1-0.2 . \mu \mathrm{g} \cdot \mathrm{kg}^{-1} \cdot \mathrm{min}^{-}$ 1 , propofol 3-6 mg $\mathrm{kg}^{-1} \cdot \mathrm{h}^{-1}$, combined with inhalation of 0.5-2 MAC sevoflurane. Sufentanil 0.05-0.1 $\mu \mathrm{g}$ $\cdot \mathrm{kg}^{-1}$ will be administered intermittently in accordance with the demand for analgesia. The mean arterial pressure and $\mathrm{HR}$ will be maintained within $30 \%$ of the baseline value. The patient's axillary temperature will be maintained at $36-37^{\circ} \mathrm{C}$ throughout the operation. During anesthesia, $A B P, \mathrm{P}_{\mathrm{ET}} \mathrm{CO}_{2}$, peak inspiratory pressure $(\mathrm{PIP})$, and plateau pressure $\left(\mathrm{P}_{\text {plat }}\right)$ will be monitored. All anesthetics will be discontinued at the end of the surgery.

\title{
Study protocol
}

Patients will be intravenously infused with the label-covered trial agent after $5 \mathrm{~min}$ of pre-medication. Lung ultrasound scans will be performed at three points in each patient: immediately after radial artery puncture (T1), 15 min after tracheal intubation (T2), and before extubation (T3). Three arterial blood samples will be obtained through radial artery puncture at each time point $(T 1, T 2, T 3)$, and blood gas measurements will be performed immediately using a standard technique (ABL800).

Agent preparation method: S-ketamine group (Group S): a $0.25 \mathrm{mg} \cdot \mathrm{kg}--1$ dose of S-ketamine will be diluted with normal saline to $5 \mathrm{~mL}$ in an opaque 5 -ml syringe. Normal saline group (group N): $5 \mathrm{~mL}$ of normal saline. Both will be labeled as "study agents"

\section{Pulmonary ultrasonography}

A well-trained blinded anesthesiologist will perform all lung ultrasound scans using SonoSite M-Turbo with a 5-12 MHz linear transducer. Patients will be scanned in the supine position following the lung ultrasound method described by Sun et al.[39]. Briefly, six regions will be scanned in each hemithorax. Scans 1 and 2 will be performed in the clavicle midline, scans 3 and 4 will be performed in the axillary midline, and the intercostal scans (Scans 5-6) will be performed at the fifth and sixth intercostal spaces in the posterior axillary line. A linear probe will be placed parallel to the ribs, and the intercostal space of each region will be scanned sequentially from right to left, cranial to caudal, and anterior to posterior within 2 min.

The video clips of each region will be stored on a portable hard drive for offline analysis. Scoring criteria of Blines: B-lines will be categorized into four grades $(0-3)$. B0 normally ventilated areas with lung sliding sign: Aline, and isolated $B$-lines ( $<3)$; B1; mild reduction in lung ventilation/moderate loss of lung tissue gasification: $B$ lines ( $\geq 3$ ) with clear boundary, regular distribution, and spacing $\geq 7 \mathrm{~mm}$; or irregular, clear interval; B2: severe reduction in lung ventilation/severe lung gasification: multiple coalescent B-lines spaced $\leq 3 \mathrm{~mm}$ and continuous fusion; B3: atelectasis/pulmonary consolidation: tissue-like signs, fragment signs, and bronchial inflation signs appear.

\section{Outcome measurement}

\author{
Primary outcome: Total LUS at T2
}


Secondary outcomes: LUS in 6 chest regions (scans 1-6) at T2; total LUS at T3; arterial blood gas analysis results $\left(\mathrm{PaCO}_{2}, \mathrm{PaO}_{2}\right)$ and $\mathrm{PaO}_{2} / \mathrm{FiO}_{2}$ at $\mathrm{T} 2$ and $\mathrm{T} 3, \mathrm{P}_{\text {plat }}$ and dynamic lung compliance (Cdyn) [Cdyn = tidal volume/(PIP-PEEP)] at T2 and T3; the incidence of postoperative complications associated with S-ketamine at $2 \mathrm{~h}$ and $24 \mathrm{~h}$ after surgery (visual impairment, dizziness, pathological irritability, nightmares, and hallucinations); the incidence of PPCs at $2 \mathrm{~h}$ and $24 \mathrm{~h}$ after surgery (the clinical outcome definitions of PPCs will adopt European joint taskforce guidelines published in 2015[40]. These include respiratory infection, respiratory failure, atelectasis, pleural effusion, pneumothorax, bronchospasm, aspiration pneumonia, pulmonary edema, ARDS, tracheobronchitis, pulmonary edema, exacerbation of pre-existing lung disease, pulmonary embolism)

\section{Other indicators to be recorded}

(1) Baseline value:

General information of patients: age, height, weight, BMI, smoking history, ASA, type of operation, complications, types, and severity of complications.

Anesthesia-related variables: duration of apnea during artificial airway establishment (recorded by stopwatch), laryngoscope insertion times, liquid volume (from entering the room to T2)

Intraoperative indicators: total LUS at T1; arterial blood gas analysis results $\left(\mathrm{PaCO}_{2}, \mathrm{PaO}_{2}\right)$ and $\mathrm{PaO}_{2} / \mathrm{FiO}_{2}$ at $\mathrm{T} 1$; mechanical ventilation parameters (tidal volume, ventilation frequency, and $\mathrm{FiO}_{2}$ ).

(2) Other indicators

Vital signs (pulse, $\mathrm{BP}, \mathrm{HR}$, and $\mathrm{SpO}_{2}$ ) at each time point $(\mathrm{T} 1, \mathrm{~T} 2$, and $\mathrm{T} 3$ ) 
Table 1

The schedule of enrollment, interventions, and assessments.

\begin{tabular}{|c|c|c|c|c|c|c|c|}
\hline \multicolumn{8}{|c|}{ STUDY PERIOD } \\
\hline \multirow[b]{2}{*}{ TIMEPOINT } & \multirow{2}{*}{$\begin{array}{l}\text { Enrollment } \\
\text { Day - } 1\end{array}$} & \multirow{2}{*}{$\begin{array}{l}\text { Allocation } \\
\text { Day } 0\end{array}$} & \multicolumn{5}{|c|}{ Postallocation } \\
\hline & & & $\begin{array}{l}\text { T1 (after } \\
\text { radial } \\
\text { artery } \\
\text { puncture) }\end{array}$ & $\begin{array}{l}15 \text { min } \\
\text { after } \\
\text { intubation } \\
\text { (T2) }\end{array}$ & $\begin{array}{l}\text { Before } \\
\text { extubation } \\
\text { (T3) }\end{array}$ & $\begin{array}{l}2 \mathrm{~h} \\
\text { after } \\
\text { surgery }\end{array}$ & $\begin{array}{l}24 \mathrm{~h} \\
\text { after } \\
\text { surgery }\end{array}$ \\
\hline \multicolumn{8}{|c|}{ ENROLLMENT } \\
\hline $\begin{array}{l}\text { Eligibility } \\
\text { screen }\end{array}$ & \multicolumn{7}{|l|}{$x$} \\
\hline $\begin{array}{l}\text { Informed } \\
\text { consent }\end{array}$ & \multicolumn{7}{|l|}{$x$} \\
\hline Randomization & \multicolumn{7}{|c|}{$x$} \\
\hline Allocation & & $x$ & & & & & \\
\hline \multicolumn{8}{|c|}{ INTERVENTIONS } \\
\hline [Group S] & & & $x$ & & & & \\
\hline \multicolumn{8}{|l|}{$\begin{array}{l}\text { S-ketamine } \\
\text { group }\end{array}$} \\
\hline [Group N] & & & $x$ & & & & \\
\hline \multicolumn{8}{|l|}{$\begin{array}{l}\text { Normal saline } \\
\text { group }\end{array}$} \\
\hline \multicolumn{8}{|c|}{ OUTCOME ASSESSMENT } \\
\hline [Total LUS] & & & $\mathrm{x}$ & $※$ & $x$ & & \\
\hline $\begin{array}{l}\text { [LUS of } 6 \text { chest } \\
\text { regions] }\end{array}$ & & & & $\mathrm{x}$ & & & \\
\hline $\begin{array}{l}{\left[\mathrm{PaCO}_{2}, \mathrm{PaO}_{2}\right.} \\
\text { and } \\
\left.\mathrm{PaO}_{2} / \mathrm{FiO}_{2}\right]\end{array}$ & & & $x$ & $x$ & $x$ & & \\
\hline $\begin{array}{l}\text { [P } P_{\text {plat }} \text { and } \\
\text { Cdyn] }\end{array}$ & & & & $x$ & $x$ & & \\
\hline $\begin{array}{l}\text { [Incidence of } \\
\text { postoperative } \\
\text { complications } \\
\text { associated S- } \\
\text { ketamine] }\end{array}$ & & & & & & $x$ & $x$ \\
\hline $\begin{array}{l}\text { [Incidence of } \\
\text { PPCs] }\end{array}$ & & & & & & $x$ & $x$ \\
\hline
\end{tabular}


LUS: lung ultrasound scores

$\mathrm{P}_{\text {plat }}$ : plateau pressure

Cdyn: dynamic lung compliance

PPCs: postoperative pulmonary complications

※primary outcome

\section{Dissemination}

The study protocol was registered at ClinicalTrials.gov (identified as NCT04745286). The results will be available and disseminated to all participants, investigators, and healthcare providers in the form of summary documents, presentations on the Internet. The data set analyzed in the current study will be obtained from the corresponding author upon reasonable request.

\section{Sample size calculation and statistical analysis}

According to previous studies, the incidence of atelectasis is approximately $90 \%$ in patients pre-oxygenated with $100 \%$ oxygen within a few minutes after anesthesia[1, 2, 5, 35]. In addition, the accuracy of lung ultrasound for the assessment of anesthesia-induced atelectasis was 88\%[41]. We assume that the preoperative administration of S-ketamine can reduce the incidence of atelectasis by $30 \%$, and the probability of atelectasis being detected by ultrasound will reach a level of about $52 \%$. According to https://www.cnstat.org/samplesize/4/, a study population of 50 cases in each group will be estimated to provide $80 \%$ power with a significance level a of 0.05 , allowing for a dropout rate of $10 \%$.

Statistical analysis will be performed using SPSS 24.0. Values will be expressed as mean \pm standard deviation ( $\overline{\mathrm{x}} \pm \mathrm{s})$, median and interquartile range (IQR), and percentages according to different distribution types. After testing for normality of continuous variables, the Student's $t$-test or the Mann-Whitney U-test will be used for comparison between and within groups, as appropriate. Categorical variables will be analyzed using the Chi-square test or Fisher's exact test. All statistical analyses in our study will be conducted with a bilateral test, and statistical significance will be set at $P<0.05$. We will exclude patients whose primary data are missing.

\section{Discussion}

In this prospective randomized controlled trial, we intend to verify whether using a small-dose S-ketamine before induction can alleviate atelectasis $15 \mathrm{~min}$ after tracheal intubation under general anesthesia, and to further study the effect of low-dose S-ketamine on PPCs. To our knowledge, to date, this is the only randomized clinical trial of preoperative agents on atelectasis. This will possibly provide a simple and feasible new method on agent prevention and direct clinical evidence for anesthesiologists to conduct intraoperative lung protection strategies. We will use the new non-invasive lung ultrasound technology as a guide for the diagnosis of atelectasis, which has many advantages, such as not being restricted by time or venue, and without radiation. In addition, to analyze the effects of the intervention on gas exchange $\left(\mathrm{PaCO}_{2}\right.$, 
$\mathrm{PaO}_{2}$, and $\left.\mathrm{PaO}_{2} / \mathrm{FiO}_{2}\right)$ in detail, we performed blood gas measurements at three-time points $(\mathrm{T} 1, \mathrm{~T} 2, \mathrm{~T} 3)$. There are limitations in this trial: (1) We will study only the effects of a small dose of ketamine on anesthesia-induced atelectasis, but will not compare the effects of different concentrations of ketamine; (2) ultrasound assessment depends on the operator and reviewer, which may cause subjective bias. However, in order to reduce this bias, all the researchers involved in the pulmonary assessment will be blinded to the allocated information, and all intercostal spaces will be carefully scanned to avoid missing any abnormal findings. In addition, the ultrasound images for each lung region will be analyzed by two experienced radiologists; (3) this is a single-center trial and may have selection bias.

\section{Abbreviations}

PPCs: postoperative pulmonary complications

$\mathrm{V}_{\mathrm{A}} / \mathrm{Q}$ : ventilation/perfusion

PEEP: positive end-expiratory pressure

LUS: lung ultrasound scores

IRB: Institutional Review Board

ASA: American Society of Anesthesiologists

BP: blood pressure

HR: heart rate

$\mathrm{SpO}_{2}:$ pulse oxygen saturation

ABP: arterial blood pressure

$\mathrm{P}_{\mathrm{ET}} \mathrm{CO}_{2}$ : end-tidal carbon dioxide partial pressure

PIP: peak inspiratory pressure

$\mathrm{P}_{\text {plat: }}$ Plateau pressure

Cdyn: dynamic lung compliance

CRF: case report form

Pl: principal investigator

\section{Declarations}

\section{Ethics approval and privacy protection}


The trial was approved by the IRB of Beijing Tiantan Hospital, Capital Medical University (approval number: KY 2019-005-05). Before surgery, when the patients meet the eligibility criteria, the researcher will explain the study plan and all potential risks to the participants and their authorized surrogates. Then, the participants will be required to sign an informed consent before enrollment. Throughout the research process, participants would be free to withdraw their consent or discontinue participation at any time without any restrictions. The reasons and corresponding circumstances will be recorded in the case report form (CRF). Participants will be asked their data can be used in a specific research project after they have signed the informed consent form. This trial will not involve the collection of biological specimens for storage.

The relevant private information of the involved patients, such as name, home address, and contact phone number, will be kept confidential. The information paper will be locked in a safety box and the electronic information will be stored in an encrypted electronic database with restricted access by the principal investigator $(\mathrm{PI})$ who will sign the confidential disclosure agreement. Data and information will not be printed or transmitted to any auxiliary media and will record the date and time when team members accessed the database. Each patient will be assigned a unique study identifier that will only appear on the data and documents during evaluation or statistical analysis. In addition, all original records, such as informed consent and CRF, will be destroyed according to hospital standards.

\section{Trial status}

The protocol version is 1.0 , October 10,2020 . The current status is patient enrolment.

\section{Availability of the data}

The CRF will be completed by a blinded researcher, and the ultrasound equipment will be calibrated uniformly. Every 30 cases completed in the experiment will be checked by the Data Monitoring Committee and stored in the security database of the Department of Anesthesiology, Beijing Tiantan Hospital, Capital Medical University. The study protocol is available on Clinicaltrial.gov (identifier: NCT04745286), and the research results, analyzed data sets, etc., can be made available upon reasonable request from the corresponding author.

\section{Data motoring}

An external independent physician who will not be involved in the study will provide monitoring, including verifying the progress of the study and all observational data. Quality control will be performed at all stages of data processing. We do not have any interim analysis plan because the intervention risk in this study is low, and it is a short-term trial.

\section{Plans for communicating important protocol amendments to relevant parties (e.g., trial participants, ethical committees)}

Any deviations from the protocol will be recorded and reported to the corresponding regulatory organization. The PI will revise the protocol according to the record and update the new version on the clinical trial registration website. The revised protocol amendments have been sent to the investigators. 


\section{Harms}

The anesthesia schemes of the patients enrolled in this project have been clinically verified for a long time and will be completed by experienced doctors. Therefore, it will not cause any additional harm to the patients. Hence, there are no compensation provisions. If there are serious adverse events related to the study agent, they will be reported to the PI and recorded in the study database. Then, the blinded method will be broken if necessary.

\section{Funding}

This trial was supported by a grant from the Beijing Dongcheng District Outstanding Talent Funding Project (No. 2019DCT-M-17), and the Research and Cultivation Plan of the Beijing Municipal Administration of Hospital (PX 2017011). The funders played no part in the study design, management, analysis, or interpretation of the data.

\section{Declaration of interest}

The author states that there is no relevant conflict of interest

\section{Acknowledgments}

We sincerely thank the Ultrasound Department of Beijing Tiantan Hospital for their support in the study design and training of investigators, as well as the assistance and cooperation of doctors and nurses in the operating room of the Beijing Tiantan Hospital.

\section{Authors' contributions}

Xu Jin and Wei Xiong conceived and designed the study. Bin Liu contributed to the study coordination. Lu Li was responsible for registration and ethical applications. Lanxin Qiao contributed to patient recruitment, Yi Liang and Yaxin Wang were responsible for clinical anesthesia, and Di Zhang is responsible for data collection. Di Zhang, Yi Liang and Di Bao contributed equally to this work and are co-first authors. Xu Jin revised and modified the protocol and the article. All named authors have read the authorship guidelines of the Trials and have agreed to its publication.

\section{- Authors' information (optional)}

- DZ\#, Department of Anesthesiology, Beijing Tiantan Hospital, Capital Medical University, Beijing, China.

- YL, Department of Anesthesiology, Beijing Tiantan Hospital, Capital Medical University, Beijing, China.

- DB\#, Department of Anesthesiology, Beijing Tiantan Hospital, Capital Medical University, Beijing, China.

- WX, Department of Anesthesiology, Beijing Tiantan Hospital, Capital Medical University, Beijing, China.

- LL, Department of Anesthesiology, Beijing Tiantan Hospital, Capital Medical University, Beijing, China.

- YW, Department of Anesthesiology, Beijing Tiantan Hospital, Capital Medical University, Beijing, China.

- BL, Department of Anesthesiology, Beijing Tiantan Hospital, Capital Medical University, Beijing, China.

- XJ*, Department of Anesthesiology, Beijing Tiantan Hospital, Capital Medical University, Beijing, China. 


\section{References}

1. Lundquist, $\mathrm{H}$., et al., CT-assessment of dependent lung densities in man during general anaesthesia. Acta radiologica (Stockholm, Sweden : 1987), 1995. 36(6): p. 626-32.

2. Brismar, B., et al., Pulmonary densities during anesthesia with muscular relaxation-a proposal of atelectasis. Anesthesiology, 1985. 62(4): p. 422-8.

3. Hedenstierna, G. and H. Rothen, Atelectasis formation during anesthesia: causes and measures to prevent it. Journal of clinical monitoring and computing, 2000. 16: p. 329-35.

4. Hedenstierna, G., et al., Oxygenation Impairment during Anesthesia: Influence of Age and Body Weight. Anesthesiology, 2019. 131(1): p. 46-57.

5. Duggan, M. and B. Kavanagh, Pulmonary atelectasis: a pathogenic perioperative entity. Anesthesiology, 2005. 102(4): p. 838-54.

6. Hedenstierna, G. and H. Rothen, Respiratory function during anesthesia: effects on gas exchange. Comprehensive Physiology, 2012. 2(1): p. 69-96.

7. Mazo, V., et al., Prospective external validation of a predictive score for postoperative pulmonary complications. Anesthesiology, 2014. 121(2): p. 219-31.

8. Zhu, C., et al., Effects of intraoperative individualized PEEP on postoperative atelectasis in obese patients: study protocol for a prospective randomized controlled trial. Trials, 2020. 21(1): p. 618.

9. Tusman, G., et al., Lung recruitment and positive end-expiratory pressure have different effects on $\mathrm{CO} 2$ elimination in healthy and sick lungs. Anesthesia and analgesia, 2010. 111(4): p. 968-77.

10. Nicholson, D., et al., Postoperative pulmonary function in coronary artery bypass graft surgery patients undergoing early tracheal extubation: a comparison between short-term mechanical ventilation and early extubation. Journal of cardiothoracic and vascular anesthesia, 2002. 16(1): p. 27-31.

11. Hedenstierna, G. and L. Edmark, Mechanisms of atelectasis in the perioperative period. Best practice \& research. Clinical anaesthesiology, 2010. 24(2): p. 157-69.

12. Duggan, M. and B. Kavanagh, Atelectasis in the perioperative patient. Current opinion in anaesthesiology, 2007. 20(1): p. 37-42.

13. Woo, S., D. Berlin, and J. Hedley-Whyte, Surfactant function and anesthetic agents. Journal of applied physiology, 1969. 26(5): p. 571-7.

14. Gommers, D., et al., Exogenous surfactant therapy increases static lung compliance, and cannot be assessed by measurements of dynamic compliance alone. Critical care medicine, 1993. 21(4): p. 567-74. 
15. Hedenstierna, G. and L. Edmark, Effects of anesthesia on the respiratory system. Best practice \& research. Clinical anaesthesiology, 2015. 29(3): p. 273-84.

16. Ostermann ME, K.S., Seiferling RA, Sibbald WJ. , Sedation in the intensive care unit: a systematic review. JAMA 2000. 283:1451-9.

17. Roytblat, L., et al., Ketamine attenuates the interleukin-6 response after cardiopulmonary bypass. Anesthesia and analgesia, 1998. 87(2): p. 266-71.

18. Yang, Z., Z. Chen, and X. Jiang, [Effects of subanesthetic dose of ketamine on perioperative serum cytokines in orthotopic liver transplantation]. Nan fang yi ke da xue xue bao = Journal of Southern Medical University, 2006. 26(6): p. 802-4, 817.

19. Yu, M., et al., Effects of ketamine on pulmonary inflammatory responses and survival in rats exposed to polymicrobial sepsis. Journal of pharmacy \& pharmaceutical sciences : a publication of the Canadian Society for Pharmaceutical Sciences, Societe canadienne des sciences pharmaceutiques, 2007. 10(4): p. 434-42.

20. Otis, D., et al., Role of pulmonary surfactant in airway closure: a computational study. Journal of applied physiology (Bethesda, Md. : 1985), 1993. 75(3): p. 1323-33.

21. Taniguchi, T., K. Shibata, and K. Yamamoto, Ketamine inhibits endotoxin-induced shock in rats. Anesthesiology, 2001. 95(4): p. 928-32.

22. Kawasaki, C., et al., Ketamine isomers suppress superantigen-induced proinflammatory cytokine production in human whole blood. Canadian journal of anaesthesia = Journal canadien d'anesthesie, 2001. 48(8): p. 819-23.

23. Yu, Y., et al., Ketamine reduces NFkappaB activation and TNFalpha production in rat mononuclear cells induced by lipopolysaccharide in vitro. Annals of clinical and laboratory science, 2002. 32(3): p. $292-8$.

24. Shaked, G., et al., Ketamine improves survival and suppresses IL-6 and TNFalpha production in a model of Gram-negative bacterial sepsis in rats. Resuscitation, 2004. 62(2): p. 237-42.

25. Mazar, J., et al., Involvement of adenosine in the antiinflammatory action of ketamine. Anesthesiology, 2005. 102(6): p. 1174-81.

26. Dale, O., et al., Does intraoperative ketamine attenuate inflammatory reactivity following surgery? A systematic review and meta-analysis. Anesthesia and analgesia, 2012. 115(4): p. 934-43.

27. Braun, S., et al., Ketamine induces apoptosis via the mitochondrial pathway in human lymphocytes and neuronal cells. British journal of anaesthesia, 2010. 105(3): p. 347-54.

28. Lange, M., et al., [Role of ketamine in sepsis and systemic inflammatory response syndrome]. Der Anaesthesist, 2006. 55(8): p. 883-91. 
29. Hofbauer Roland; Moser, D., Ketamine significantly inhibits the transmigration of leukocytes through endothelial cell monolayers Critical Care Medicine, January 1999. 27:p 165A,

30. Copetti, R., et al., Lung ultrasound in respiratory distress syndrome: a useful tool for early diagnosis. Neonatology, 2008. 94(1): p. 52-9.

31. Haller, J., et al., Sonographic evaluation of the chest in infants and children. AJR. American journal of roentgenology, 1980. 134(5): p. 1019-27.

32. Lichtenstein, D., Ultrasound examination of the lungs in the intensive care unit. Pediatric critical care medicine : a journal of the Society of Critical Care Medicine and the World Federation of Pediatric Intensive and Critical Care Societies, 2009. 10(6): p. 693-8.

33. Acosta, C., et al., Accuracy of transthoracic lung ultrasound for diagnosing anesthesia-induced atelectasis in children. Anesthesiology, 2014. 120(6): p. 1370-9.

34. Lichtenstein, D., et al., The "lung pulse": an early ultrasound sign of complete atelectasis. Intensive care medicine, 2003. 29(12): p. 2187-2192.

35. Edmark, L., et al., Optimal oxygen concentration during induction of general anesthesia. Anesthesiology, 2003. 98(1): p. 28-33.

36. Östberg, E., et al., Positive End-expiratory Pressure and Postoperative Atelectasis: A Randomized Controlled Trial. Anesthesiology, 2019. 131(4): p. 809-817.

37. Jang, Y., et al., Effect of regular alveolar recruitment on intraoperative atelectasis in paediatric patients ventilated in the prone position: a randomised controlled trial. British journal of anaesthesia, 2020. 124(5): p. 648-655.

38. Strandberg, A., et al., Atelectasis during anaesthesia and in the postoperative period. Acta anaesthesiologica Scandinavica, 1986. 30(2): p. 154-8.

39. Sun, L., et al., Lung ultrasound evaluation of incremental PEEP recruitment maneuver in children undergoing cardiac surgery. Pediatric pulmonology, 2020. 55(5): p. 1273-1281.

40. Lumb, A.M.a.A.B., Postoperative pulmonary complications. British Journal of Anaesthesia, 2017.118 (3): p. 317-34 (2017.

41. Cecilia M.Acosta, M.D., Gustavo A.Maidana, ,Accuracy of Transthoracic Lung Ultrasound for Diagnosing Anesthesia-induced Atelectasis in Children. Anesthesiology, 2014. 120:1370-9.

\section{Figures}




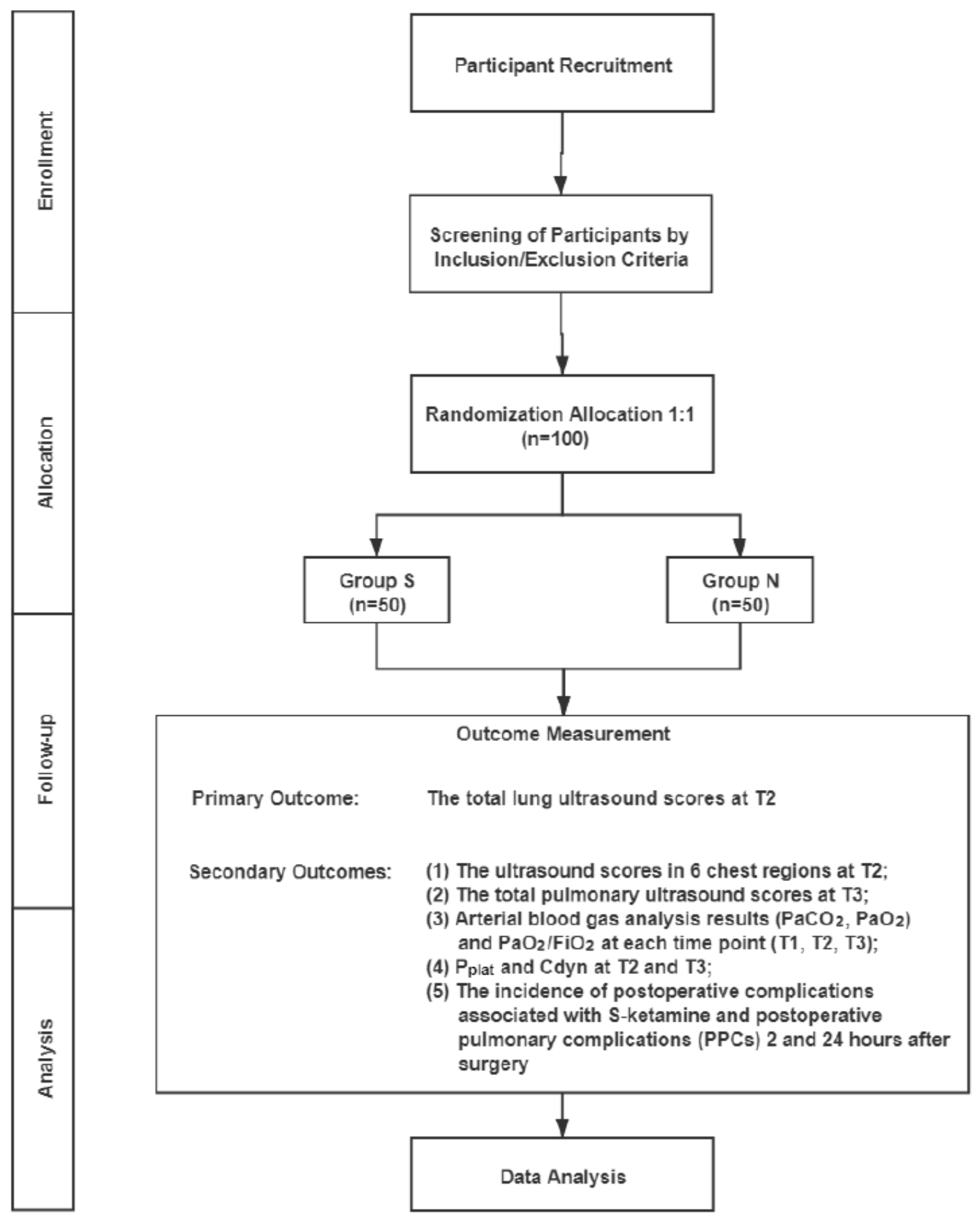

Figure 1

Flow diagram of the consolidated standards of reporting trials (CONSORT). Group S: S-ketamine group; Group N: Normal saline group T1: After radial artery puncture T2: $15 \mathrm{~min}$ after tracheal intubation T3: Before extubation

\section{Supplementary Files}


This is a list of supplementary files associated with this preprint. Click to download.

- spiritchecklist.docx 